Combinatorics, Probability and Computing (2007) 00, 000-000. (C) 2007 Cambridge University Press DOI: $10.1017 /$ S0000000000000000 Printed in the United Kingdom

\title{
Teasing apart two trees
}

\author{
M. A. STEEL ${ }^{1}$ and L. A. SZÉKELY ${ }^{2}$ \\ $\dagger$ \\ ${ }^{1}$ Biomathematics Research Centre, Department of Mathematics and Statistics, \\ University of Canterbury, Christchurch, New Zealand. Email: m.steel@math.canterbury.ac.nz \\ 2 Department of Mathematics, University of South Carolina \\ Columbia SC, USA. Email: szekely@math.sc.edu
}

\begin{abstract}
A widely-studied model for generating binary sequences is to 'evolve' them on a tree according to a symmetric Markov process. We show that under this model distinguishing the true (model) tree from a false one is substantially "easier" (in terms of the sequence length needed) than determining the true tree. The key tool is a new and near tight Ramsey-type result for binary trees.
\end{abstract}

\section{Introduction}

In this paper we investigate the reconstruction of trees from binary sequences that have been generated on the tree by a simple Markov model. Such processes are widely-studied in molecular genetics, and in other areas of applied probability (including broadcasting, information theory and statistical physics $[7,13,16])$. The questions we consider are motivated in part by the concept of NP in computational complexity. In that setting one may have short proofs to the correctness of answers to decision problems that require substantial search. For example, it is hard to find a Hamiltonian cycle in a graph, but if we are given the solution, it is easy to verify. As Erdős, Steel, Székely and Warnow pointed out in [6], reconstructing trees from randomly-evolved sequences has two distinct complexity problems. One is the sequence length: what is the minimum sequence length

$\dagger$ We thank the NZIMA (Maclaurin Fellowship) for supporting this research. The second author was supported in part by NSF contracts Nr. 0072187, 0302307, 070111 and NIH NIGMS 1 R01 GM07899101. 
to do phylogeny reconstruction with probability near 1, using any algorithm? The other is the more conventional computational complexity of the problem. In this paper we focus on the first problem, the sequence length. In particular we initiate the study of the following natural question: is it possible using few observed patterns from the true tree, to test an input tree, i.e. reject any false input tree with high probability, and to accept an input true tree with high probability? If yes, can this be done in polynomial time? We make the following progress in this direction: using few observed patterns from the true tree, if we are shown the true tree and a false one, we can tell with high probability which one is true, and we make this decision in polynomial time.

A widely-studied and applied approach for generating sequences is to 'evolve' them on a binary tree according to some Markov process. The simplest such model involves just two character states (and so generates binary sequences) and has symmetric transition matrices on all edges of the tree. This model is referred to as the CFN (Cavender-FarrisNeyman) model in molecular biology, although in other arenas it has been referred to as the 'symmetric binary channel' and the 'symmetric 2-state Poisson model' (we define it more precisely in the next Section). The CFN model thus provides a simple model for the evolution of purine-pyrimidine sequences on phylogenetic trees. Apart from its relative tractability, a major reason for investigating this simple model is that phenomena shown for the CFN model often extend to more realistic models of sequence evolution (the CFN model itself is now rarely used in molecular biology, but most results established here should extend to more widely-used models, though with more complex arguments).

In phylogenetic tree reconstruction using a model such as the CFN model, the input consists of corresponding (similar, but not identical) purine-pyrimidine sequences from $n$ taxa. One assumes that $n$ taxa are identified with the $n$ leaves of a binary tree describing the true evolutionary history of the taxa, and that every site (i.e. position) in the observed sequences developed according to the same CFN model, and independently of each other. The goal is to use these sequences to reconstruct the underlying binary tree describing the true evolutionary history.

Formally, the phylogeny reconstruction problem $(P h y R e P)$ requires the reconstruction of the topology of the model binary tree from $k$ independent sites (observations of character states at the leaves). Since sites develop randomly, at best PhyReP can be solved with high probability (whp).

We have shown in [6] that if $|X|=n$ and $n \rightarrow \infty$, then $k=\Omega(\log n)$ sites are needed to return the true underlying tree with probability at least $\frac{1}{2}+\epsilon$ with either a deterministic algorithm or with a randomized algorithm whose random bits are independent from the random events on the $\mathrm{CFN}$ tree.

We also showed in [6] that PhyReP is possible whp for all model trees, when $k$ is a certain polynomial of $n$; is possible for some model trees, when $k$ is a logarithmic function of $n$; and is possible for almost all model trees (either in the uniform random binary tree model or in the Yule-Harding model), when $k$ is a certain polylogarithmic function of $n$. 
More recent work by E. Mossel and colleagues [4, 12] has established further instances for which logarithmic dependence of $k$ on $n$ suffices for accurate tree reconstruction and cases for which polynomial dependence is necessary. Sequence length requirements for accurate tree reconstruction are not only of theoretical interest, but also a topical issue in molecular systematics (eg. $[2,15])$.

Following Popperian epistemology, it would be highly desirable to develop methods to refute claimed phylogenetic trees, especially if refutation is 'simpler' than solving PhyReP. Indeed, this was our motivation when we initiated the study of the sequence length needed to solve PhyReP whp.

This paper introduces a new decision problem and a test to solve it, which is a step towards a refutation technique. The phylogeny distinguishing problem $(P h y D i P)$ is the following: suppose we are given $k$ sites that have been generated on a CFN model tree, and a set of two trees, one is the model tree, and another one. Determine whp which tree was the model tree.

In Theorem 3.1 we provide a test, which is a polynomial time randomized algorithm, that solves PhyDiP from constant length sequences under mild assumption on the CFN model. Constant length contrasts the $k=\Omega(\log n)$ requirement for PhyReP, i.e. PhyDiP is simpler than PhyReP in terms of sequence length needed.

An evolutionary biologist might wish to compare the maximum likelihood scores of the two input trees for a PhyDiP test. However this approach faces two hurdles: (1) computing maximum likelihood scores of trees is a problem of unknown complexity (and which is generally solved by heuristic hill-climbing techniques) and (2) it is not clear if maximum likelihood prefers the true tree with high probability from very short sequences [22]. Accordingly, the test we describe here involves an approach that is different from maximum likelihood estimation.

Normally, one verifies that two binary phylogenetic trees are different by exhibiting 4 taxa that span different 4-leaf subtrees in the trees. The key-and also hardestcomponent of our test is a new, asymmetric criterion to verify that two binary phylogenetic trees are different (Theorem 4.2).

The proof of the main result also requires the existence of a certain novel kind of phylogenetic tree reconstruction method for 4-leaf CFN trees (Lemma 5.2). Roughly speaking, it must return the true tree with near 1 probability from $O(1)$ length sequences, if mutation probabilities are somewhat restricted, but even if the restriction fails, it should pick the true tree with at least some fixed positive probability, say $1 / 4$. We conjecture that some natural phylogeny reconstruction methods, like maximum likelihood estimation or Buneman's 4-point condition also satisfy this property, but to formulate a proof looked so inconvenient that we instead developed a new method, for which these properties can be more easily established. 
We close the summary with an open problem. What is the sequence length needed to solve whp the phylogeny decision problem $(P h y D e P)$ : suppose we are given $k$ sites that have been generated on a CFN model tree, and an input tree. Determine whp whether or not the input tree was the model tree. The sequence length complexity of PhyDeP clearly falls between the sequence length complexities of PhyReP and PhyDeP.

To describe our results more precisely we first provide some terminology concerning trees and random processes on them.

\section{Definitions and technical preliminaries}

In a tree, vertices of degree 1 are called leaves and the edges adjacent to them are called leaf edges. Other edges are called internal edges. A tree is binary, if all vertices have degree 1 or 3 . Consider a set $X$ of labels. These labels are usually called taxa or species in biology. A phylogenetic $X$-tree is a tree, in which leaves are identified with elements of $X$. We will regard two phylogenetic $X$-trees as being identical, if there is a graph isomorphism between them, which in addition, if restricted to $X$, is the identity function of $X$. The distance between two vertices in a tree is the number of edges on the unique path connecting them. A cherry is a pair of leaves of distance two apart.

For a phylogenetic $X$-tree $\mathcal{T}$ and subset $Y$ of $X$, let $\mathcal{T}(Y)$ denote the minimal subtree that connects the leaves in $Y$, and let $\mathcal{T} \mid Y$ denote the associated phylogenetic $Y$-tree obtained from $\mathcal{T}(Y)$ by suppressing vertices of degree 2. Given an edge $e$ of $\mathcal{T} \mid Y$ let subdiv $(e)$ denote the number of degree two vertices of $\mathcal{T}(Y)$ that were suppressed in forming $e$. For a phylogenetic tree $\mathcal{T}$ and an interior edge $e$ let $\mathcal{T} / e$ denote the phylogenetic tree obtained by contracting edge $e$. Notice that if $\mathcal{T}$ is binary then $\mathcal{T} / e$ is not binary.

For a phylogenetic $X$-tree $\mathcal{T}$ and an edge $e$ of it, we speak about the split induced by $e$. The split is an equivalence relation on $X$ induced by "being in one component" after the deletion of $e$.

In particular, if $\mathcal{T}(Y)$ is binary and $Y$ has four elements, say $Y=\{a, b, c, d\}$ (sometimes called a quartet), then $\mathcal{T} \mid Y$ has a single internal edge, and the removal of this edge from $\mathcal{T} \mid Y$ partitions the vertices of $Y$ into two -element classes by connectivity. The 3 possible partitions are called quartet splits and denoted as follows:

$$
a b \mid c d \quad \text { or } a c \mid b d \text { or } a d \mid b c .
$$

If $\mathcal{T}|Y=a b| c d$ we say that $\mathcal{T}$ displays $a b \mid c d$, written $\left.a b\right|_{\mathcal{T}} c d$.

We now describe a model for the evolution of binary sequences on a tree. This model has been described by various authors (and in a range of disciplines including molecular biology, information theory, and physics; for references see $[7,16])$. Here we refer to this model as the CFN model (short for 'Cavender-Farris-Neyman model'). 
Suppose we have two character states, 0 and 1, and a phylogenetic $X$-tree $\mathcal{T}$. The CFN model assigns probabilities to the patterns of state of the elements of $X$ as follows. Let us be given $0<f \leq g<1 / 2$ and associate a number $p_{e}\left(f \leq p_{e} \leq g\right)$ with the edge $e$ called the substitution probability. Let $\xi_{e}$ denote a random indicator variable associated to edge $e$ with $\mathbb{P}\left[\xi_{e}=1\right]=p_{e}$, and assume the $\xi_{e}$ 's are independent. Fix any vertex $v$. For every vertex $u$, there is a unique path denoted path $(u, v)$ in $\mathcal{T}$. Define

$$
\operatorname{state}(u)=\operatorname{state}(v)+\sum_{e \in \operatorname{path}(u, v)} \xi_{e} \bmod 2 .
$$

One approach to define pattern probabilities is to assign state 0 and 1 to $v$ with probability $1 / 2$, and then compute the probabilities of all possible state patterns of leaves under (2.1). An essentially equivalent approach is to consider as pattern the classes of the equivalence relation "being in the same state" for the elements of $X$. A pattern of this second kind is the identification of two complementary patterns of the first kind. Let the pattern $\sigma$ denote a state assignment to every leaf. Let $\mathcal{P}_{\sigma}$ denote the probability of observing pattern $\sigma$ under the first approach. Then, if $\bar{\sigma}$ is the probability of the bitwise complementary pattern, then $\mathcal{P}_{\sigma}=\mathcal{P}_{\bar{\sigma}}$, and if $[\sigma]$ denotes the class of $\sigma$ after the identification of complementary patterns, then simply $\mathcal{P}_{[\sigma]}=2 \mathcal{P}_{\sigma}$.

The probability $p$ that the endpoints of a path $u w$ in a CFN tree $\mathcal{T}$ are in different states is nicely related to the substitution probabilities of edges of the $u w$-path:

$$
p=\frac{1}{2}\left(1-\prod_{e \in \operatorname{path}(u, w)}\left(1-2 p_{e}\right)\right) .
$$

Formula (2.2) is well-known, and is easy to prove by induction. Formula (2.2) also shows that the substitution probability of a path is not less than the smallest transition probability on its edges. It is well-known and easy to see ([20]) that (1) changing the location of $v$ in $\mathcal{T}$, or (2) substituting a path with internal vertices of degree 2 with a single edge in a CFN tree, and assigning to the new edge a transition probability according to (2.2) does not change the probability distribution of patterns.

Usually $k$ independent observations, called sites, are sampled from a binary CFN tree $\mathcal{T}$.

In (most of) this paper we study problems where the bounds $f, g$ are fixed, and we let $n \rightarrow \infty$. However many of the results generalize to provide results where these quantities are allowed to depend on $n$, provided the dependence on $n$ does not exceed a certain critical value. 


\section{The main result}

Our main result is the following.

Theorem 3.1. Suppose we have a true CFN binary phylogenetic $X$-tree $\mathcal{F}_{1}$ with transition mechanism $0<f \leq p_{e}<\gamma<1 / 8$ and another binary phylogenetic $X$-tree $\mathcal{F}_{2}$. For every $\epsilon^{\prime}>0$ there is a test, which from the input of $K=K\left(\epsilon^{\prime}, f, \gamma\right)$ sites evolved on the true tree, and the input of the 2-element set $\left\{\mathcal{T}_{1}, \mathcal{T}_{2}\right\}$ (where $\left\{\mathcal{T}_{1}, \mathcal{T}_{2}\right\}=\left\{\mathcal{F}_{1}, \mathcal{F}_{2}\right\}$ ), tells which input tree is the true tree with probability $\geq 1-\epsilon^{\prime}$. Moreover, this test can be realized by a randomized algorithm that is polynomial time in $|X|$.

The crucial point is that $K\left(\epsilon^{\prime}, f, \gamma\right)$ is not dependent on $|X|$. The fact that this fixed number of sites is independent of the number of vertices of the tree is in contrast to the $\Omega(\log n)$ lower bound for the sequence length required for abstract phylogeny reconstruction, mentioned in the Introduction.

The proof of Theorem 3.1 is given in Section 5 and relies on an unusual characterization of when two binary trees are different. This is a Ramsey type result proved in Theorem 4.1, and the proof uses a number of estimates for a number of novel extremal problems on binary trees. Many of those may be interesting on their own. First we make some further remarks concerning Theorem 3.1.

\section{Remarks}

- The condition $0<f \leq p_{e}$ is easily seen to be necessary for Theorem 3.1, as is an upper bound of the form $p_{e} \leq \gamma<1 / 2$. However the additional requirement that $\gamma<1 / 8$ deserves some discussion. The value $1 / 8$ allows us to use some nice properties of a known phylogenetic approach (based on maximum parsimony). This raises an interesting question, namely whether Theorem 3.1 could be improved by allowing a larger bound on $\gamma$. It seems possible that one may be able, with more work, to replace the value $\frac{1}{8}$ by a value closer to (or equal to) $\frac{1}{2}\left(1-\frac{1}{\sqrt{2}}\right)$ which would be the largest possible value for which the Theorem 3.1 can hold (by results in [7]).

- The problem of selecting the true tree from a set of two trees could clearly be generalized to selecting the true tree from an arbitrary set of trees. Provided the set has given size (independent of $n$ ) a multiple pairwise comparison argument shows that (an analogue of) Theorem 3.1 holds. However if the size of the set of trees from which the true tree is to be selected whp grows with $n$ then an elementary counting-style argument shows that the required sequence length must also grow with $n$. 


\section{A Ramsey type result for trees}

The following Ramsey type combinatorial result has important statistical implications for distinguishing between two trees using $\mathrm{O}(1)$ sites. The result is Ramsey type in the following sense: no matter how different binary phylogenetic $X$-trees one tries to make, one still finds some regularity.

Theorem 4.1. For any two different binary phylogenetic $X$-trees, $\mathcal{T}_{1}, \mathcal{T}_{2}$, at least one of the following must occur:

(i) $\mathcal{T}_{1}$ and $\mathcal{T}_{2}$ share a cherry, or

(ii) there exists a 4-element subset $Y$ of $X$ so that

- $\mathcal{T}_{1}$ and $\mathcal{T}_{2}$ induce different quartet splits on $Y$, and

- each leaf edge e of $\mathcal{T}_{1} \mid Y$ has at most four subdividing vertices in $\mathcal{T}_{1}(Y)$, i.e. we have $\operatorname{subdiv}(e) \leq 4$.

Using this theorem, one can readily obtain the following result (Theorem 4.2) which is more conveniently formulated for application in the next Section.

Theorem 4.2. For any two different binary phylogenetic $X$-trees, $\mathcal{T}_{1}, \mathcal{T}_{2}$ there exists a subset $Y$ of $X$ so that:

(i) $\mathcal{T}_{1}\left|Y \neq \mathcal{T}_{2}\right| Y$.

(ii) There are interior edges $e_{1}, e_{2}$ of $\mathcal{T}_{1} \mid Y$ and $\mathcal{T}_{2} \mid Y$ respectively so that

$$
\left(\mathcal{T}_{1} \mid Y\right) / e_{1}=\left(\mathcal{T}_{2} \mid Y\right) / e_{2} .
$$

(iii) among the edges of $\mathcal{T}_{1} \mid Y$, the four edges adjacent to $e_{1}$ each have at most four subdividing vertices in $\mathcal{T}_{1}(Y)$, and other edges different from $e_{1}$ are not subdivided at all.

Such a subset $Y$ can be found in polynomial time from $\mathcal{T}_{1}, \mathcal{T}_{2}$.

Note the asymmetry of $\mathcal{T}_{1}$ and $\mathcal{T}_{2}$ in the theorems above. We do not see any obvious reason why these theorems should work, even for some large $t$ replacing $\operatorname{subdiv}(e) \leq 4$ with $\operatorname{subdiv}(e) \leq t$. It is possible that the condition $\operatorname{subdiv}(e) \leq 4$ in these theorems might be able to be weakened to $\operatorname{subdiv}(e) \leq 3$ (but not weakened further to $\operatorname{subdiv}(e) \leq 2$ for which counterexamples are known).

We show first how Theorem 4.1 implies Theorem 4.2.

Proof of Theorem 4.2 (assuming Theorem 4.1).

If $\mathcal{T}_{1}$ and $\mathcal{T}_{2}$ have no cherries in common, then the implication is obvious and a quartet guaranteed in Theorem 4.1 can be found by checking all quartets. Otherwise recursively 
contract common cherries: if $\mathcal{T}_{1}$ and $\mathcal{T}_{2}$ share a cherry - say $a, b$ - then replace the cherry both in $\mathcal{T}_{1}$ and $\mathcal{T}_{2}$ with a single new leaf $(a b)$, to obtain $\mathcal{F}_{1}$ and $\mathcal{F}_{2}$ with $X_{F}=$ $X \backslash\{a, b\} \cup\{(a b)\}$. Repeat this until the case with $\mathcal{T}_{1}$ and $\mathcal{T}_{2}$ have no cherries in common applies. Find an appropriate quartet as above, and then undo the contractions to find the required set $Y \subset X$. Clearly, the steps described can all be implemented in polynomial time.

In order to prove Theorem 4.1 we first present a series of lemmas.

Suppose we are given a binary phylogenetic $X$-tree $\mathcal{T}$ with a positive edge weighting $w$, and assume a linear order is provided on $X$. Define the representative quartet of an internal edge $e$ of $\mathcal{T}$ to be the quartet (four elements of $X$ ) defined as follows. $e$ is adjacent to four edges, $e_{i}, i=1,2,3,4$. For every $i$, consider the shortest (in weighted distance) path containing $e_{i}$ and some leaf, but not $e$ itself. Select from the leaves realizing the minimum weighted distance from $e_{i}$ (as above) the smallest regarding the linear order given of $X$. The four leaves obtained in this way make the representative quartet of $e$. In [6] the following result was established:

Lemma 4.3. Any binary phylogenetic $X$-tree $\mathcal{T}$, with any positive edge weighting, and with any linear order given on $X$, is determined by the set of splits of the representative quartets of $\mathcal{T}$ in the following sense: if all these splits are present in a binary phylogenetic $X$-tree $\mathcal{T}^{\prime}$, then $\mathcal{T}=\mathcal{T}^{\prime}$.

The proof of the following result (Lemma 4.4) is straightforward, and omitted.

Lemma 4.4. Assume that $\mathcal{T}$ is a binary tree and leaves $u, v$ form a cherry in $\mathcal{T}$. If vertices $c, d$ are within distance 4 from $u$ (and hence from $v$ ), then $c$ and $d$ are within distance 4 from each other.

Next we introduce a family of equivalence relations on $X$ which will be useful throughout this section. Given a phylogenetic $X$-tree $\mathcal{T}$ and a positive integer $r$ define a graph on $X$ by joining $i \in X$ and $j \in X$, if their distance in $\mathcal{T}$ is at most $r$. Consider the transitive closure of this relation, the equivalence relation $\sim_{r}$. We will generally use $r=6$ except in Lemma 4.5 and 4.6 , where we will also consider $r=4$.

Lemma 4.5. Consider a binary $X$-tree $\mathcal{T}$ with $m=m(\mathcal{T})=|X| \geq 2$ and the equivalence relation $\sim_{4}$. Then the equivalence relation has at most $m / 2$ classes.

Proof. The claim is easy to check for $2 \leq m \leq 6$. Assume that $m \geq 7$ and apply induction on $m$, based on the shape of the ending of a longest path. The tree $\mathcal{T}$ must have 
a longest path with an ending falling into the 4 cases shown on Fig. $1 . \mathcal{T}_{i}(i=1,2,3,4)$ refer to the cases, and $\mathcal{T}_{i}^{\prime}$ is the result of truncation as indicated by the curve on Fig. 1.

Let $\#_{r}(\mathcal{T})$ denote the number of $\sim_{r}$ classes of the leaf set of the tree $T$. We have

$$
\#_{4}\left(\mathcal{T}_{1}\right)=\#_{4}\left(\mathcal{T}_{1}^{\prime}\right) \leq m\left(\mathcal{T}_{1}^{\prime}\right) / 2=m\left(\mathcal{T}_{1}\right) / 2-1
$$

by induction. We claim $\#_{4}\left(\mathcal{T}_{2}\right) \leq \#_{4}\left(\mathcal{T}_{2}^{\prime}\right)+1$. (Indeed, think about the top leaves of $\mathcal{T}_{2}^{\prime}$ as

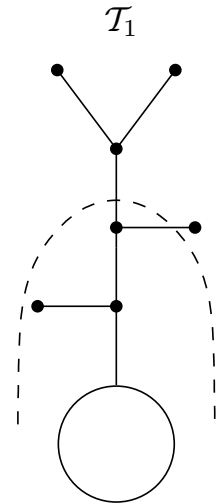

$\mathcal{T}_{1}^{\prime}$

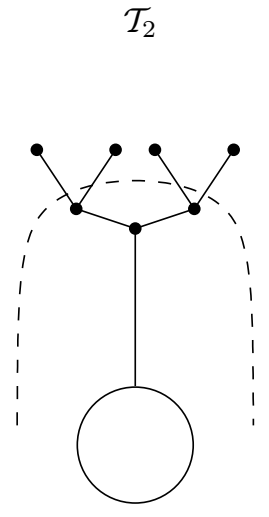

$\mathcal{T}_{2}^{\prime}$

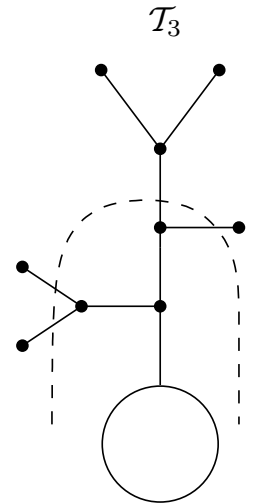

$\mathcal{T}_{3}^{\prime}$
$\mathcal{T}_{4}$

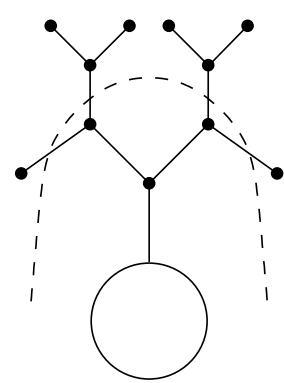

$\mathcal{T}_{4}^{\prime}$

Figure 1. Ending of a longest path in a binary tree.

$u, v$ in Lemma 4.4. This lemma implies that merging $u$ and $v$ into $\mathcal{T}_{2}$ and giving up their leafness cannot split any $\sim_{4}$ class of $\mathcal{T}_{2}^{\prime}$ into parts. The top four leaves of $\mathcal{T}_{2}$ belong to the same $\sim_{4}$ class of $\mathcal{T}_{2}$.) Continue with $\#_{4}\left(\mathcal{T}_{2}^{\prime}\right)+1 \leq m\left(\mathcal{T}_{2}^{\prime}\right) / 2+1=\left(m\left(\mathcal{T}_{2}\right)-2\right) / 2+1=$ $m\left(\mathcal{T}_{2}\right) / 2$. Similarly,

$$
\#_{4}\left(\mathcal{T}_{3}\right) \leq \#_{4}\left(\mathcal{T}_{3}^{\prime}\right)+1 \leq m\left(\mathcal{T}_{3}^{\prime}\right) / 2+1=\left(m\left(\mathcal{T}_{3}\right)-3\right) / 2+1=m\left(\mathcal{T}_{3}\right) / 2-1 / 2,
$$

and

$$
\#_{4}\left(\mathcal{T}_{4}\right) \leq \#_{4}\left(\mathcal{T}_{4}^{\prime}\right)+1 \leq m\left(\mathcal{T}_{4}^{\prime}\right) / 2+1=\left(m\left(\mathcal{T}_{4}\right)-4\right) / 2+1=m\left(\mathcal{T}_{4}\right) / 2-1
$$

Fig. 2 shows that Lemma 4.5 is basically tight.

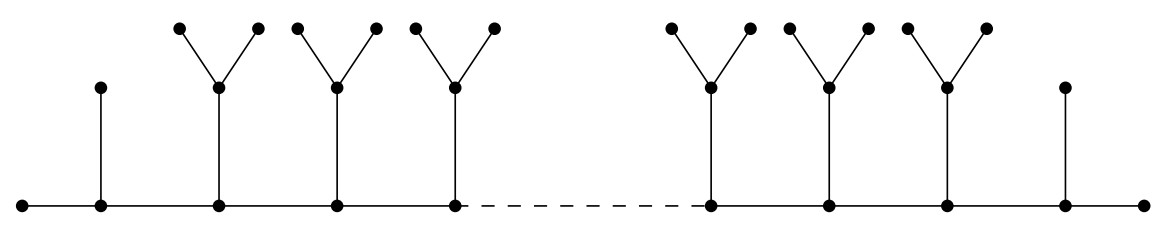

Figure 2. $m(\mathcal{T})=2 k+4$ and $\#_{4}(\mathcal{T})=k$. 
Lemma 4.6. Assume that $\mathcal{T}$ is a binary $X$-tree on $n \geq 4$ leaves, on which the equivalence relation $\sim_{6}$ has $k$ classes. Then $\mathcal{T}$ has at least $2 k$ cherries.

Proof. The proof uses induction on $n$, with base case $n=4$. We consider two cases: (a) every leaf of $\mathcal{T}$ is in a cherry; (b) $\mathcal{T}$ has a leaf not in a cherry. We will denote by $\operatorname{ch}($. the number of cherries, and by $n($.$) the number of leaves in a tree.$

For case (a) replace $\mathcal{T}$ by $\mathcal{T}^{*}$ contracting every cherry to a single leaf vertex. Then $\operatorname{ch}(\mathcal{T})=n\left(\mathcal{T}^{*}\right) \geq 2 \#_{4}\left(\mathcal{T}^{*}\right)$ by Lemma 4.5 , and obviously $\#_{4}\left(\mathcal{T}^{*}\right)=\#_{6}(\mathcal{T})$, and so the result holds.

In case (b) assume that $x$ is a leaf in $\mathcal{T}$, but not in a cherry. Then $\mathcal{T}$ must have at least four other leaves. Introduce a new leaf name $x^{\prime}$ and create two new trees $\mathcal{T}_{1}$ and $\mathcal{T}_{2}$ from $\mathcal{T}$ as indicated on Fig. 3.

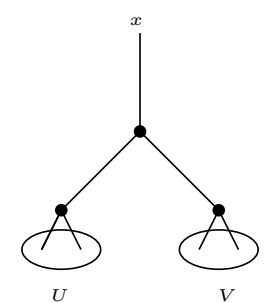

$\mathcal{T}$

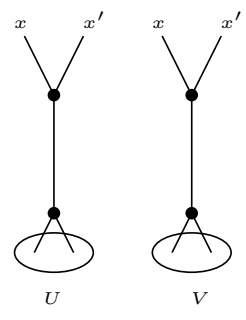

$\mathcal{T}_{1} \quad \mathcal{T}_{2}$

Figure 3. Creating two new trees from $\mathcal{T}$ in case (b).

Both $\mathcal{T}_{1}$ and $\mathcal{T}_{2}$ have at least four leaves and the induction hypothesis applies to them. Simple counting shows that

$$
\operatorname{ch}(\mathcal{T})=\operatorname{ch}\left(\mathcal{T}_{1}\right)+\operatorname{ch}\left(\mathcal{T}_{2}\right)-2 .
$$

A straightforward case analysis, based on how many of the sets $U, V$ intersects the $\sim_{6^{-}}$ class of $x$ in $\mathcal{T}$, yields that for all cases

$$
\#_{6}(\mathcal{T})=\#_{6}\left(\mathcal{T}_{1}\right)+\#_{6}\left(\mathcal{T}_{2}\right)-1
$$

Using (4.1), the hypothesis for $\mathcal{T}_{1}$ and $\mathcal{T}_{2}$, and (4.2), we obtain

$$
\operatorname{ch}(\mathcal{T})=\operatorname{ch}\left(\mathcal{T}_{1}\right)+\operatorname{ch}\left(\mathcal{T}_{2}\right)-2 \geq 2 \#_{6}\left(\mathcal{T}_{1}\right)+2 \#_{6}\left(\mathcal{T}_{2}\right)-2=2 \#_{6}(\mathcal{T}) .
$$

This completes the proof of Lemma 4.6.

Proof of Theorem 4.1. Note that Theorem 4.1 holds trivially for $n=4$. Assume that Theorem 4.1 is not true and let $\mathcal{T}_{1}$ and $\mathcal{T}_{2}$ be two phylogenetic $X$-trees that do not satisfy the conclusions of this theorem. In particular this means that $|X|>4$ and the following holds:

$\mathcal{T}_{1}$ and $\mathcal{T}_{2}$ have no cherries in common, 
and

$$
\left(Y \subset X,|Y|=4 \text { and } \operatorname{subdiv}(e) \leq 4 \text { for all leaf edges } e \text { of } \mathcal{T}_{1} \mid Y\right) \Longrightarrow \mathcal{T}_{1}\left|Y=\mathcal{T}_{2}\right| Y
$$

Let $A_{1}, A_{2}, \ldots, A_{k}$ denote the classes of $\sim_{6}$ on the tree $\mathcal{T}_{1}$ (the equivalence relation was defined just prior to Lemma 4.5). We claim that

$$
\text { for } i=1,2, \ldots, k, \mathcal{T}_{1} \mid A_{i} \text { is isomorphic to } \mathcal{T}_{2} \mid A_{i},
$$

and for all $l=1,2$ and for all $1 \leq i<j \leq k$, there is a split of $\mathcal{T}_{l}$ (depending on $i, j$ ), such that

$$
A_{i} \text { and } A_{j} \text { are contained in different sides of the split of } \mathcal{T}_{l} \text {. }
$$

Once we have established (4.5) and (4.6), we have two binary $X$-trees $\mathcal{T}_{1}, \mathcal{T}_{2}$ on $n$ leaves that do not share any cherry (4.3), and satisfy both (4.5) and (4.6). This means that from $\mathcal{T}_{l}(l=1,2)$ one can remove $k-1$ edges and suppress all vertices of degree 2 , to obtain the isomorphic binary forests $\mathcal{F}=\left\{\mathcal{T}_{1} \mid A_{i}: i=1,2, \ldots, k\right\}$ and $\mathcal{F}=\left\{\mathcal{T}_{2} \mid A_{i}: i=1,2, \ldots, k\right\}$. Apply now Lemma 4.6 to $\mathcal{T}_{1}$ to find $2 k$ cherries. None of the cherries are divided between the $\sim_{6}$ equivalence classes, and therefore they are still cherries in the forest $\mathcal{F}$. However, from forest $\mathcal{F}$ we can construct the binary tree $\mathcal{T}_{2}$ by repeating the following step $k-1$ times: insert vertices of degree 2 into some edges of the two current components, and join the vertices of degree 2 with a new edge. (If a current component comprises a single vertex, then there is no insertion of a degree 2 vertex, just join the new edge to this single vertex.) Every inserted edge kills at most 2 cherries present, so we can kill at most $2(k-1)$ cherries of $\mathcal{F}$. Therefore, $\mathcal{T}_{1}$ and $\mathcal{T}_{2}$ have a cherry in common, contradicting (4.3).

Thus all that remains is to establish (4.5) and (4.6). This is what comprises the remainder of the proof.

Proof of (4.5). If $\left|A_{i}\right| \leq 3$, the conclusion holds automatically. Therefore we may assume $\left|A_{i}\right| \geq 4$. We are going to establish that the set of all representative quartet splits of $\mathcal{T}_{1} \mid A_{i}$ are also quartet splits of $\mathcal{T}_{2} \mid A_{i}$. From here, Lemma 4.3 implies $\mathcal{T}_{1}\left|A_{i}=\mathcal{T}_{2}\right| A_{i}$. Consider now an internal edge $f \in E\left(\mathcal{T}_{1} \mid A_{i}\right)$. Edge $f$ is the result of contraction of a path $\pi_{f}$ in $\mathcal{T}_{1}\left(A_{i}\right)$. Since $A_{i}$ was defined as a class of an equivalence relation defined on the vertex set of $\mathcal{T}_{1}$ by the closure of the relation "exists a path of length at most 6 between two leaves", we can think of $\mathcal{T}_{1}\left(A_{i}\right)$ being covered with paths of length at most 6 , going from leaf to leaf, and reaching from every leaf to every other leaf in a few steps.

To describe the basic quartet split construction, consider four paths in $\mathcal{T}_{1}\left(A_{i}\right)$, leaving the endvertices $u, v$ of the path $\pi_{f}$, internally disjoint from $\pi_{f}$ and each other, connecting to the four closest leaves in $\mathcal{T}_{1}\left(A_{i}\right)$. Let these leaves be $a, b$ at one end $u$ and $c, d$ at the other end $v$.

Let $Y=\{a, b, c, d\}$. Observe the following property of the basic quartet split construction: $d_{\mathcal{T}_{1}}(a, b) \leq 6$ or paths of length at most 6 passing through $f$ connect $a(b)$ to some leaves. It is easy to see in both cases that $d_{\mathcal{T}_{1}}(a, u) \leq 5$ and $d_{\mathcal{T}_{1}}(b, u) \leq 5$. A similar argument yields $d_{\mathcal{T}_{1}}(c, v) \leq 5$ and $d_{\mathcal{T}_{1}}(d, v) \leq 5$. Since we just showed that in $\mathcal{T}_{1} \mid Y$ for 
any leaf edge $e$ we have subdiv(e) $\leq 4$, (4.4) implies that $\mathcal{T}_{1}\left|Y=\mathcal{T}_{2}\right| Y$, in other words $a b \mid c d$ is displayed by $\mathcal{T}_{2}$. Next, we show that the quartet splits of the basic quartet split construction in $\mathcal{T}_{1}\left(A_{i}\right)$ (that a fortiori generates the same quartet splits in $\mathcal{T}_{1} \mid A_{i}$ ) provide all representative quartet splits of $\mathcal{T}_{1} \mid A_{i}$ under some weighting of the edges of $\mathcal{T}_{1} \mid A_{i}$, and therefore we are in a position to apply Lemma 4.3. Namely, use for the weight of an edge of $\mathcal{T}_{1} \mid A_{i}$ the number of edges in the path representing this edge in $\mathcal{T}_{1}\left(A_{i}\right)$. Indeed, take now any path from $e \in E\left(\mathcal{T}_{1} \mid A_{i}\right)$ in a direction to a closest leaf in $\mathcal{T}_{1} \mid A_{i}$. If the weight of this path is $w$, then there is a path from $\pi_{e}$ in $\mathcal{T}_{1}\left(A_{i}\right)$ to a leaf of distance $w$, and this must be a closest path in $\mathcal{T}_{1}\left(A_{i}\right)$. (Any linear ordering of $X$ suffices.) This establishes (4.5).

Proof of (4.6). If $\left|A_{i}\right|=1$ or $\left|A_{j}\right|=1$, then there is nothing to prove. We may assume $\left|A_{i}\right| \geq 2$ and $\left|A_{j}\right| \geq 2$. Take $a, b \in A_{i}$ and $c, d \in A_{j}$ distinct leaves. First we are going to show that $\mathcal{T}_{1}$ displays the quartet split $a b \mid c d$, that is:

$$
\left.a b\right|_{\mathcal{T}_{1}} c d
$$

Formula (4.7) is clearly equivalent to the fact that the $a b$ and $c d$ paths in $\mathcal{T}_{1}$ are disjoint. Assume for the contrary, that $a b$ and $c d$ paths in $\mathcal{T}_{1}$ intersect, say edge $f$ is in the intersection.

Recall that the $a b$ path can be covered in $\mathcal{T}_{1}$ with a sequence of paths of length $\leq$ 6 , each connecting vertices from $A_{i}$, and similarly, the $c d$ path can be covered in $\mathcal{T}_{1}$ with a sequence of paths of length $\leq 6$, each connecting vertices from $A_{j}$. Some of these $\leq 6$-paths contain $f$, and it follows that there are $a^{\prime}, b^{\prime} \in A_{i}$ with $d_{\mathcal{T}_{1}}\left(a^{\prime}, b^{\prime}\right) \leq 6$, and $c^{\prime}, d^{\prime} \in A_{j}$ with $d_{\mathcal{T}_{1}}\left(c^{\prime}, d^{\prime}\right) \leq 6$, such that the $a^{\prime} b^{\prime}$ and $c^{\prime} d^{\prime}$ paths in $\mathcal{T}_{1}$ intersect. Therefore $\left.a^{\prime} c^{\prime}\right|_{\mathcal{T}_{1}} b^{\prime} d^{\prime}$ or $\left.a^{\prime} d^{\prime}\right|_{\mathcal{T}_{1}} b^{\prime} c^{\prime}$. In the first case, $d_{\mathcal{T}_{1}}\left(a^{\prime}, c^{\prime}\right)+d_{\mathcal{T}_{1}}\left(b^{\prime}, d^{\prime}\right)<12$, which implies that $a^{\prime}, c^{\prime}, b^{\prime}, d^{\prime}$ are all in one equivalence class. The second case provides a similar contradiction. Now formula (4.7) implies that in $\mathcal{T}_{1}$ any two paths connecting $A_{i}$ to $A_{j}$ intersect. Recall the Helly property of trees: if a collection of subtrees of a tree pairwise intersect, then they all intersect (see [9], Ex. 6.16, p. 41). Therefore all these paths intersect in an edge or a vertex of $\mathcal{T}_{1}$. A simple case analysis shows that a trivalent vertex cannot be such a common intersection, if it is not part of an intersection edge. This establishes (4.6) for $\mathcal{T}_{1}$.

For $\mathcal{T}_{2}$, we still have $\left|A_{i}\right| \geq 2$ and $\left|A_{j}\right| \geq 2$. Let $a, b \in A_{i}$ with $d_{\mathcal{T}_{1}}(a, b) \leq 6$ and $c, d \in A_{j}$ with $d_{\mathcal{T}_{1}}(c, d) \leq 6$. These with (4.7) means that for $Y=\{a, b, c, d\}$ for any leaf edge $e$ in $\mathcal{T}_{1} \mid Y$ we have $\operatorname{subdiv}(e) \leq 4$; therefore (4.4) gives us that

$$
\left.a b\right|_{\mathcal{T}_{2}} c d
$$

We now use the following quartet inference rule: If any binary $X$-tree displays the quartet splits $a x \mid c d$ and $b x \mid c d$ then it necessarily displays the quartet split $a b \mid c d([3,5])$. Applying this inference rule we see that (4.8) holds for $a, b \in A_{i}$ and $c, d \in A_{j}$ with no distance condition as well.

This completes the proof of (4.6) and thereby of Theorem 4.1. 


\section{Proof of Theorem 3.1}

In order to describe the test with the properties claimed in Theorem 3.1 we first discuss ancestral state reconstruction. Assume that we have a binary phylogenetic $X$-tree. Subdivide an edge with a vertex $r$, and use this $r$ and a character associated with it to build a CFN model with leafs tates assigned, as in (2.1). We call this a rooted CFN tree. The ancestral state reconstruction problem is as follows: from the states assigned to the leaves, try to identify the state of $r$ (the tree and the location of $r$ is known). The Fitch-Hartigan algorithm (see [16], p. 90) recursively assigns state sets to vertices of the rooted CFN tree, starting with leaves, going towards the root $r$. If the state of a leaf is $x$ ( $x=0$ or 1$)$, then let the state set of the leaf be $\{x\}$. If both sons of a vertex already have state sets $M$ and $N$ assigned, then assign to this vertex state set $M \cap N$, if $M \cap N \neq \emptyset$, otherwise $M \cup N$. (The original Fitch-Hartigan algorithm was designed to evaluate the parsimony score of a given tree, and upon parsing the tree once more from root towards the leaves it also constructs a most parsimonious reconstruction of states on the tree. We use the algorithm here for a different purpose). We will use the following simple algorithm (called randomized $F H$ ) for ancestral state reconstruction: if the root has a singleton state set, we reconstruct for ancestral state the element of this singleton set; and if the root has a doubleton state set, we select the ancestral state by tossing a fair coin.

The proof of the following result is given in Section 6 .

Lemma 5.1. Consider any rooted binary phylogenetic tree, $\mathcal{T}$, evolve a single site under the CFN model starting with an arbitrary character state in the root, such that $p(e)<1 / 2$ for each edge e of $\mathcal{T}$. Then the probability that the randomized Fitch-Hartigan algorithm correctly reconstructs the root state is

(i) strictly greater than $\frac{1}{2}$;

(ii) at least $\frac{1}{2}+\Delta_{g}$, where

$$
\Delta_{g}=\frac{\sqrt{(1-4 g)(1-8 g)}}{2(1-2 g)^{2}},
$$

provided that $p_{e} \leq g<1 / 8$ for each edge e of $\mathcal{T}$.

The test that works for Theorem 3.1 is the following. Use Theorem 4.2 to find a set $Y \subset X$ for the ordered pair $\mathcal{T}_{1}, \mathcal{T}_{2}$. Consider the tree $\mathcal{T}_{1} \mid Y$. Remove the edge $e_{1}$ and 4 edges adjacent to it in $\mathcal{T}_{1} \mid Y$. We are left with 4 rooted phylogenetic trees, $\mathcal{C}_{i}$ with root $r_{i}$. (Note that $\mathcal{C}_{i}$ is present in $\mathcal{T}_{1}$ itself as one side of an edge.) Let the leaf set of $\mathcal{C}_{i}$ be $X_{i}$. Observe that removing the edge $e_{2}$ and 4 edges adjacent to it in $\mathcal{T}_{2} \mid Y$ results in the very same $\mathcal{C}_{i}, X_{i}$, and $r_{i}$. Assume without loss of generality that $r_{1} r_{2}$ and $r_{3} r_{4}$ are separated by $e_{1}$ in $\mathcal{T}_{1} \mid Y$, but $r_{1} r_{3}$ and $r_{2} r_{4}$ are separated by $e_{2}$ in $\mathcal{T}_{2} \mid Y$.

Consider now an input site developed on the true tree $\mathcal{F}_{1}$. For $i=1,2,3,4$, use the 
randomized Fitch-Hartigan algorithm to reconstruct the ancestral state of $r_{i}$ using the character states in $X_{i}$ and the rooted tree $\mathcal{C}_{i}$. For a $K$ (to be specified later), repeat the ancestral state reconstruction above for $K$ independent sites. We have 4 sequences of length $K$ associated with $r_{i}, i=1,2,3,4$. Feed these sequences as an input into a tree reconstruction which meets the specification of the following Lemma.

Lemma 5.2. Let $\delta$ be any positive number that is less than $\frac{1}{3}$. Then there exists a tree reconstruction method $M\left(=M_{\delta}\right)$ for 4-leaf binary phylogenetic trees equipped with the CFN model, which has the following two properties:

(i) Suppose that each pendant edge e of the true 4-leaf tree has $0<f \leq p_{e} \leq g<1 / 2$, and we have $0<f \leq p_{e}<1 / 2$ on the central edge. Then for any $\epsilon>0$, there exists a constant $k=k(\epsilon, f, g)$ number so that the probability the method $M$ returns the true tree from at least $k$ i.i.d. sites, is at least $1-\epsilon$.

(ii) $M$ correctly reconstructs each true tree $\mathcal{T}$ with probability at least $\delta$ under any $C F N$ transition mechanism on $\mathcal{T}$, from any number of sites.

(Section 7 contains the proof of Lemma 5.2. We conjecture that many tree reconstruction methods, including the Buneman four-point condition and maximum likelihood estimation, actually satisfy these conditions.) We select $\delta=1 / 4, \epsilon=0.01, f$ and $\gamma$ from the conditions of Theorem 3.1, $g$ from

$$
g=\frac{1}{2}\left(1-(1-2 \gamma)^{5}\left(1-2\left(\frac{1}{2}-\Delta_{\gamma}\right)\right)\right),
$$

and we set $M$ for these numbers. Apply $M$ to $K / k(\epsilon, f, g)$ disjoint $k(\epsilon, f, g)$-tuples of the $K$ sites defined by the 4 sequences associated with $r_{i}(i=1,2,3,4)$. The output of each application of $M$ is a 4-leaf tree, that we identify with one of $r_{1} r_{2}\left|r_{3} r_{4}, r_{1} r_{3}\right| r_{2} r_{4}$, $r_{1} r_{4} \mid r_{2} r_{3}$. If we get $r_{1} r_{2} \mid r_{3} r_{4}$ in at least $7 / 8$ of the $K / k(\epsilon, f, g)$ outputs of $M$, we output $\mathcal{T}_{1}$ as our guess for the true tree $\mathcal{F}_{1}$. Otherwise we output $\mathcal{T}_{2}$.

This is the test, and the remainder of this section is devoted to establishing the proof of its correctness. The proof considers two cases: either $\mathcal{T}_{1}$ is the true tree or $\mathcal{T}_{2}$ is the true tree.

We select $K=\max \left(K_{1}, K_{2}\right)$, where $K_{1}=K_{1}\left(\epsilon^{\prime}, f, \gamma\right)$ sites are sufficient when $\mathcal{T}_{1}$ is the true tree and $K_{2}=K_{2}\left(\epsilon^{\prime}, f, \gamma\right)$ sites are sufficient when $\mathcal{T}_{2}$ is the true tree (the $K_{i}$ will be specified later).

Assume first that $\mathcal{T}_{1}$ is the true tree, i.e. $\mathcal{T}_{1}=\mathcal{F}_{1}$. Then $\mathcal{C}_{i}$ has an inherited rooted CFN structure from the true tree, keeping transition probabilities from edge to edge, and $\gamma$ is an upper bound for these transition probabilities. By Lemma 5.1(ii), the randomized FitchHartigan algorithm applied to $\mathcal{C}_{i}$ reconstructs the true state of $r_{i}$ in $\mathcal{F}_{1} \mid Y$ with probability at least $\frac{1}{2}+\Delta_{\gamma}$. Let $q_{i}$ denote the probability that, for a site randomly generated under the model, the randomized Fitch-Hartigan algorithm incorrectly reconstructs the state 
of $r_{i}$. Let us now equip the 4-leaf binary tree $\mathcal{R}$ identified by $r_{1} r_{2} \mid r_{3} r_{4}$ with a transition mechanism as follows. The transition probability of the backbone edge is the transition probability on the path in $\mathcal{T}_{1}$ corresponding to $e_{1}$ in $\mathcal{T}_{1} \mid Y$; and if $w_{i}$ is the transition probability on the path in $\mathcal{T}_{1}$ corresponding to the edge connecting $r_{i}$ to $e_{1}$ in $\mathcal{T}_{1} \mid Y$, let the transition probability $p_{i}$ of the $r_{i}$ leaf edge in $\mathcal{R}$ be recomputed by

$$
p_{i}=\frac{1}{2}\left(1-\left(1-2 w_{i}\right)\left(1-2 q_{i}\right)\right) .
$$

\section{Lemma 5.3.}

(i) $\mathcal{R}$ is a CFN model tree with the transition mechanism above;

(ii) all edge transition probabilities are at least $f$ ( $f$ is from the conditions of Theorem 3.1); and all leaf edge transition probabilities are at most $g$, where $g$ is as in (5.2);

(iii) the distribution of the leaf coloration pattern of $\mathcal{R}$ is exactly the same as that of the result of the ancestral state reconstruction for $r_{i}(i=1,2,3,4)$.

Proof. For part (i) note that independence of transitions on different edges follows from the fact random events influencing transitions on edges of $\mathcal{R}$ come from disjoint edge sets of $\mathcal{T}_{1}$. It is clear that transition probabilities are in $(0, .5)$. For part (ii), the transition probabilities stay above $f$, since every edge that may come into a path had transition probability at least $f$. Clearly $g<1 / 2$ from (5.2). A leaf edge in $\mathcal{R}$ has transition mechanism which a combination of those of at most five edges of $\mathcal{T}_{1}$ (Theorem 4.2), and of ancestral site reconstruction, which errs with probability $q_{i} \leq 1 / 2-\Delta_{\gamma}$. From here, the upper bound for the combined transition probability (5.2) easily follows from (2.2) and (5.3). Part (iii) is obvious from the construction.

When we apply $M$ to a $k(\epsilon, f, g)$-tuple of sites in the test, it is no different from applying $M$ to a $k(\epsilon, f, g)$-tuple of sites of $\mathcal{R}$, according to Lemma 5.3(iii). Lemma 5.3(i),(ii) certifies that Lemma 5.2 can be used. Therefore $M$ correctly reconstructs $\mathcal{R}$ with probability at least $1-\epsilon=0.99$. We apply $M$ to $k(\epsilon, f, g)$-tuples of sites $K_{1} / k(\epsilon, f, g)$ times. The number of cases when $\mathcal{R}$ is correctly returned follows a binomial distribution. The probability of getting $\mathcal{R}$ in less than $7 / 8$ of the experiments goes to zero, as $K_{1} \rightarrow \infty$. For this case of the proof, we can take a $K_{1}=K_{1}\left(\epsilon^{\prime}, f, \gamma\right)$ which puts this probability below $\epsilon^{\prime}$.

Consider now the second case that $\mathcal{T}_{2}$ is the true tree, i.e. $\mathcal{T}_{2}=\mathcal{F}_{1}$. Then $\mathcal{C}_{i}$ has an inherited rooted CFN structure from the true tree by suppressing non-root vertices of degree 2 and computing compound transition probabilities by (2.2) for the new edges. By Lemma 5.1(i), the randomized Fitch-Hartigan algorithm applied to $\mathcal{C}_{i}$ reconstructs the true state of $r_{i}$ in $\mathcal{F}_{1} \mid Y$ with a probability $>1 / 2$. Let $q_{i}$ denote the probability that in a site the randomized Fitch-Hartigan algorithm incorrectly guessed the state of $r_{i}$. 
Let us now equip the 4-leaf binary tree $\mathcal{R}^{\prime}$ identified by $r_{1} r_{3} \mid r_{2} r_{4}$ with a CFN transition mechanism as follows. The transition probability of the backbone edge is the inherited transition probability of $e_{2}$ in $\mathcal{T}_{2} \mid Y$; and if $w_{i}$ is the inherited transition probability of the edge connecting $r_{i}$ to $e_{2}$ in $\mathcal{T}_{2} \mid Y$, let the transition probability $p_{i}$ of the $r_{i}$ leaf edge be given by (5.3) again. Now the trick comes again: it is easy to see - we skip the detailsthat for $\mathcal{R}^{\prime}$, Lemma $5.3(\mathrm{i})$, (iii) still hold. When we apply $M$ to a $k(\epsilon, f, g)$-tuple of sites in the test, it is no different from applying $M$ to a $k(\epsilon, f, g)$-tuple of sites of $\mathcal{R}^{\prime}$, according to Lemma 5.3(iii). Lemma 5.3(i) certifies that Lemma 5.2(ii) can be used. Therefore $M$ correctly reconstructs $\mathcal{R}^{\prime}$ with probability at least $1 / 4$. We apply $M$ to $k(\epsilon, f, g)$-tuples of sites $K_{2} / k(\epsilon, f, g)$ times. The number of cases when $\mathcal{R}$ is correctly returned follows binomial distribution. The probability of getting $\mathcal{R}^{\prime}$ in more than $7 / 8$ of the experiments goes to zero, as $K_{2} \rightarrow \infty$. For this case of the proof, we can take $K_{2}=K_{2}\left(\epsilon^{\prime}, f, \gamma\right)$ which puts this probability below $\epsilon^{\prime}$.

\section{Proof of Lemma 5.1}

Let $S$ (respectively $D$ ) be the probability that the randomized Fitch-Hartigan algorithm applied to a site randomly generated on $\mathcal{T}$ finds a unique state for the root of $\mathcal{T}$ without tossing a coin, and this state is the true state (resp. not the true state). Let $E$ be the probability that the randomized Fitch-Hartigan algorithm applied to a site randomly generated on $T$ finds a doubleton state set at the root, and makes a decision with tossing a coin. Note that the probability that we can reconstruct the true root state using the randomized Fitch-Hartigan algorithm is

$$
S+\frac{1}{2} E=\frac{1}{2}+\frac{1}{2}(S-D),
$$

(since $S+D+E=1$ ). We first show(assuming that the $p(e)$ 's are all less that $\frac{1}{2}$ ) that $S-D>0$ which implies that $S+\frac{1}{2} E>\frac{1}{2}$ as claimed.

To establish $S-D>0$ we will use induction on the depth $h$ of $\mathcal{T}$ (the length of the longest path from the root to any leaf). For $h=1$ the result is easily verified. Suppose it holds for all trees of depth $h$, and let $\mathcal{T}$ be a tree of depth $h+1$. Consider the two rooted subtrees of $T$ that are incident with the root of $\mathcal{T}$ - call them $\mathcal{T}_{1}$ and $\mathcal{T}_{2}$ (one may be a leaf, but this causes no problem). Let the two edges that connect their roots to the root of $\mathcal{T}$ be (respectively) $e_{1}$ and $e_{2}$, and for $i=1,2$ let $p_{i}:=p\left(e_{i}\right), q_{i}:=1-p\left(e_{i}\right)$, and by assumption we have $q_{i}-p_{i}>0$.

Now consider computing $S, D, E$ for $\mathcal{T}_{i}$ (ignoring the rest of $\mathcal{T}$ ) - call them $S_{i}, D_{i}, E_{i}-$ for $i=1,2$. Thus, for example, $S_{1}$ is the probability that the randomized Fitch-Hartigan algorithm applied to a site randomly generated on $\mathcal{T}_{1}$ finds a unique state for the root of $\mathcal{T}_{1}$ without tossing a coin, and this state is the true state. The following fundamental recursions are from [18]:

$$
S=\left(q_{1} S_{1}+p_{1} D_{1}\right)\left(q_{2} S_{2}+p_{2} D_{2}\right)+E_{1}\left(q_{2} S_{2}+p_{2} D_{2}\right)+E_{2}\left(q_{1} S_{1}+p_{1} D_{1}\right),
$$


and

$$
D=\left(p_{1} S_{1}+q_{1} D_{1}\right)\left(p_{2} S_{2}+q_{2} D_{2}\right)+E_{1}\left(p_{2} S_{2}+q_{2} D_{2}\right)+E_{2}\left(p_{1} S_{1}+q_{1} D_{1}\right) .
$$

If follows by easy algebra that

$$
S-D=\left(q_{1} q_{2}-p_{1} p_{2}\right)\left(S_{1} S_{2}-D_{1} D_{2}\right)+E_{1}\left(q_{2}-p_{2}\right)\left(S_{2}-D_{2}\right)+E_{2}\left(q_{1}-p_{1}\right)\left(S_{1}-D_{1}\right) .
$$

But each of these three terms is strictly positive, since $q_{i}>p_{i}$ and since (by induction) $S_{i}>D_{i}$ for $i=1,2$. This completes the induction step that $S-D>0$ and thereby the proof of part (i) of Lemma 5.1.

Part (ii) of Lemma 5.1 was proved in the Honours Thesis of Kahn Mason [10], under the guidance of the first author. Here we provide a shorter, more direct proof.

By a complete binary tree (of depth $h$ ) we mean a rooted binary tree, having $2^{h}$ leaves, each at distance $h$ from the root. Suppose we are given any rooted binary phylogenetic tree $\mathcal{T}$, with substitution probabilities, and for which the maximum distance of any leaf from the root is $h$. We can convert $T$ into a complete binary tree of depth $h^{\prime} \geq h$ (with associated substitution probabilities) by the following procedure. If leaf $x$ of $\mathcal{T}$ has distance $h_{x}$ from the root, then identify $x$ with the root of a complete binary tree of depth $h^{\prime}-h_{x}$, and assign a substitution probability 0 to all the new edges in this subtree. If we do this for each leaf, then the resulting tree $\mathcal{T}^{\prime}$ is a complete binary tree of depth $h^{\prime}$ and for which $0 \leq p(e) \leq g<1 / 8$. Furthermore, the randomized Fitch-Hartigan algorithm correctly reconstructs the true ancestral state of $\mathcal{T}^{\prime}$ (with substitution probabilities as specified) with exactly the same probability as it does for $\mathcal{T}$.

Now (6.2) implies that $S-D$ is a strictly decreasing function of $p_{i}$ and a strictly increasing function of $\left(S_{i}-D_{i}\right)$ (for $\left.i=1,2\right)$. Thus the $S-D$ value for $\mathcal{T}$ is at least the $S-D$ value for $\mathcal{T}^{\prime}$ with the substitution probabilities as described, and therefore at least the $S-D$ value of a complete binary tree of depth $h^{\prime}$ having substitution probability equal to $g$ on every edge. Furthermore this holds for all $h^{\prime}$ greater or equal to the maximal distance of any leaf of $\mathcal{T}$ to the root of $\mathcal{T}$. Now Theorem 3 of [19] whose proof appears in [18] (related but more general results appear in [11]) states that for the (rooted) complete binary tree of depth $h$ and substitution probability $g<\frac{1}{8}$ on every edge, the limiting value $($ as $h \rightarrow \infty)$ of $\frac{1}{2}(S-D)$ is $\Delta_{g}$. Thus $\Delta_{g}$ is a lower bound to $\frac{1}{2}(S-D)$ for $\mathcal{T}$ with its original substitution probabilities, as claimed. This completes the proof of Lemma 5.1.

\section{Proof of Lemma 5.2}

We first establish two preliminary results. The proof of the first will require the AzumaHoeffding inequality (see [1]) which states the following:

Lemma 7.1. Suppose $\mathbf{X}=\left(X_{1}, X_{2}, \ldots, X_{k}\right)$ are independent random variables taking values in any set $S$, and $L: S^{k} \rightarrow \mathbb{R}$ is any function that satisfies the condition: $\mid L(\mathbf{u})-$ 
$L(\mathbf{v}) \mid \leq t$ whenever $\mathbf{u}$ and $\mathbf{v}$ differ at just one coordinate. Then,

$$
\mathbb{P}[L(\mathbf{X})-\mathbb{E}[L(\mathbf{X})] \geq \lambda] \quad \text { and } \quad \mathbb{P}[L(\mathbf{X})-\mathbb{E}[L(\mathbf{X})] \leq-\lambda] \leq \exp \left(-\frac{\lambda^{2}}{2 t^{2} k}\right) .
$$

Lemma 7.2. Suppose that $\hat{s}$ is the frequency counts of a multinomial distribution with $k$ trials and with expectation vector $\mathbb{E}[\hat{s}]=s$. Then, for $b>0$,

$$
\mathbb{P}\left[|s-\hat{s}|_{2}>(1+b) / \sqrt{k}\right] \leq \exp \left(-b^{2} / 4\right) .
$$

where $|\cdot|_{2}$ denotes Euclidean distance.

Proof. Consider the random variable $Y(=Y(\hat{s})):=|s-\hat{s}|_{2}$ as a function of the $k$ (independent) site patterns. Suppose one of these site patterns is changed - in which case $\hat{s}$ changes in two co-ordinates by $\frac{1}{k}$ - all other co-ordinates are unchanged. Let $\hat{s}^{\prime}$ denote this perturbation of $\hat{s}$, and $Y^{\prime}=Y\left(\hat{s}^{\prime}\right)$. Then, by the triangle inequality, $\left|Y-Y^{\prime}\right| \leq$ $\left|\hat{s}-\hat{s}^{\prime}\right|_{2}=\frac{\sqrt{2}}{k}$. Consequently, by the Azuma-Hoeffding inequality (Lemma 7.1) we have

$$
\mathbb{P}[Y-\mathbb{E}[Y]>\lambda] \leq \exp \left(-\lambda^{2} k / 4\right)
$$

Now, if $N$ denotes the number of categories of the multinomial distribution, then $\mathbb{E}[Y] \leq$ $\sqrt{\mathbb{E}\left[Y^{2}\right]}$ and

$$
\mathbb{E}\left[Y^{2}\right]=\mathbb{E}\left[\sum_{i=1}^{N}\left(\hat{s_{i}}-s_{i}\right)^{2}\right]=\sum_{i=1}^{N} \mathbb{E}\left[\left(\hat{s_{i}}-s_{i}\right)^{2}\right]=\sum_{i=1}^{N} \frac{1}{k} s_{i}\left(1-s_{i}\right)
$$

(here we use the fact that for the frequency counts of a multinomial distribution $\mathbb{E}\left[\left(\hat{s}_{i}-\right.\right.$ $\left.\left.s_{i}\right)^{2}\right]=\operatorname{Var}\left[\hat{s}_{i}\right]=\frac{1}{k} s_{i}\left(1-s_{i}\right)$.) In particular, $\mathbb{E}\left[Y^{2}\right] \leq \frac{1}{k}$ and so $\mathbb{E}[Y] \leq \frac{1}{\sqrt{k}}$. Consequently,

$$
\mathbb{P}\left[Y>\lambda+\frac{1}{\sqrt{k}}\right] \leq \exp \left(-\lambda^{2} k / 4\right)
$$

The result now follows by taking $\lambda=b / \sqrt{k}$.

Lemma 7.3. Consider the $C F N$ model on two different four taxon trees $\mathcal{T}$ and $\mathcal{T}^{\prime}$, and suppose that on $\mathcal{T}$ we have the following restriction on the substitution probabilities: for the four leaf edges

while for the central edge

$$
0 \leq p_{e} \leq g<\frac{1}{2}
$$

$$
0<f \leq p_{e} \leq \frac{1}{2}
$$

Suppose that on $\mathcal{T}^{\prime}$ the substitution probabilities are completely unrestricted-that is for this tree the only requirement for each edge $e$ is that $0 \leq p_{e} \leq \frac{1}{2}$. Let $s$ and $s^{\prime}$ be the 
vector of probabilities of site patterns produced by $\mathcal{T}$ and $\mathcal{T}^{\prime}$ (respectively) with substitution probabilities satisfying the constraints described. Then

$$
\left|s-s^{\prime}\right|_{2} \geq c f(1-2 g)^{4}
$$

for an absolute constant $c>0$ that is independent of $f$ and $g$.

Proof. Without loss of generality we may assume $X=\{1,2,3,4\}$ and that $\mathcal{T}$ is the tree $12 \mid 34$ and $\mathcal{T}^{\prime}$ is the tree $13 \mid 24$. We may regard $s$ and $s^{\prime}$ as sitting in the 7 -dimensional simplex $\Delta_{7} \subset \mathbb{R}^{8}$. Let $F$ be any real-valued differentiable function on this simplex, for which the first derivative of $F$ is bounded above on $\Delta_{7}$ in absolute value say by $M$. Then, by elementary calculus,

$$
\left|F(s)-F\left(s^{\prime}\right)\right| \leq M \sum_{i=1}^{8}\left|s_{i}-s_{i}^{\prime}\right|=M\left|s-s^{\prime}\right|_{1},
$$

where $|\cdot|_{1}$ denotes the $L_{1}-$ norm. It follows that $\left|s-s^{\prime}\right|_{2} \geq \frac{1}{M \sqrt{8}}\left|F(s)-F\left(s^{\prime}\right)\right|$.

We are going to chose a quadratic function $F$, and for such a function, a finite positive value of $M$ certainly exists. Thus it suffices to show that

$$
\left|F(s)-F\left(s^{\prime}\right)\right| \geq c^{\prime} f(1-2 g)^{4}
$$

for a constant $c^{\prime}$. Now, let $p(13 ; 24)$ be the probability leaves 1 and 3 are in different states, and leaves 2 and 4 are in different states. Thus $p(13 ; 24)$ is a sum of certain $s$ values (and also a sum of certain $s^{\prime}$ values). Similarly, let $p(13)$ be the probability that leaves 1 and 3 are in different states, and let $p(24)$ be the probability that leaves 2 and 4 are in different states. These are also linear functions of $s$ (and of $s^{\prime}$ ). Our quadratic function is $F=p(13 ; 24)-p(13) p(24)$. It is well known and easy to see that $F\left(s^{\prime}\right)=0$ (i.e. $F$ is a quadratic phylogenetic invariant for the tree $13 \mid 24$ under the CFN model which reflects the property of that model that changes across two edge-disjoint paths in the tree are statistically independent). On the other hand, algebraic manipulation shows that

$$
F(s)=p_{0}\left(1-p_{0}\right) \prod_{i=1}^{4}\left(1-2 p_{i}\right)
$$

where $p_{0}$ is the substitution probability on the central edge of $\mathcal{T}$, and $p_{i}$ is the transition probability for the leaf edge of $\mathcal{T}$ incident with $i$. Consequently, by the restrictions imposed on the $p_{e}$ values, we have $F(s) \geq f(1-f)(1-2 g)^{4}$, and so we can take $c^{\prime}=\frac{1}{2}$ in (7.2). This completes the proof.

We turn to the proof of Lemma 5.2. Denote the three trees on four taxa by $\mathcal{T}_{1}, \mathcal{T}_{2}, \mathcal{T}_{3}$. The method $M=M_{\delta}$ is as follows. Firstly let $b_{k}$ be an unbounded increasing sequence, with $\delta=\frac{1}{3}\left(1-\exp \left(-b_{1} / 4\right)\right)$ and $b_{k} / \sqrt{k} \rightarrow 0$ as $k \rightarrow \infty$.

Given $\hat{s}$ we say one of these three trees - say $\mathcal{T}_{i}$ - is 'good' (for $\hat{s}$ ) if there exist substitution probabilities (the $p_{e}$ values) for $\mathcal{T}_{i}$ for which $s\left(\mathcal{T}_{i},\left\{p_{e}\right\}\right)$ lies within Euclidean 
distance at most $\left(1+b_{k}\right) / \sqrt{k}$ from $\hat{s}$ (here $s\left(\mathcal{T}_{i},\left\{p_{e}\right\}\right)$ is the probability vector of patterns generated by $\mathcal{I}_{i}$ with parameters $\left.\left\{p_{e}\right\}\right)$.

From $\hat{s}$ construct the good trees (there may be none, one, two or three). If there are none, select one of the three trees uniformly at random. Otherwise if the set of good trees is nonempty then select one of them uniformly at random.

We claim that $M$ satisfies conditions (i) and (ii) of Lemma 5.2. Moreover, we note in passing that the running time of $M$ does not depend on $n$ (it involves a solving a numerical optimization problem on three quartet trees). We suppose without loss of generality that $\mathcal{T}_{1}$ is the true tree.

To establish condition (i) we need to specify the function $k(\epsilon, f, g)$. Given $\epsilon, f, g$ let $k(\epsilon, f, g)$ be the smallest value of $k$ for which the following two inequalities hold:

$$
\exp \left(-b_{k}^{2} / 4\right) \leq \epsilon
$$

and

$$
\frac{1+b_{k}}{\sqrt{k}}<\frac{1}{2} c f(1-2 g)^{4}
$$

where $c$ is the constant in Lemma 7.3. Suppose now that $k \geq k(\epsilon, f, g)$ and consider the event $E$ that $\mathcal{T}_{1}$ is a good tree. Then Lemma 7.2 and condition (7.3) implies that $E$ has probability at least $1-\epsilon$. Furthermore, if $s^{\prime}$ is the vector of pattern probabilities generated by one of the other trees, then by the triangle inequality we have:

$$
\left|s^{\prime}-\hat{s}\right|_{2} \geq\left|s^{\prime}-s\right|_{2}-|s-\hat{s}|_{2} \geq c f(1-2 g)^{4}-|s-\hat{s}|_{2},
$$

where the second inequality is due to Lemma 7.3. Now, conditional on event $E$, by Lemma 7.2, we have $|s-\hat{s}|_{2} \leq \frac{1+b_{k}}{\sqrt{k}}$ and so, by inequality (7.4) we have $|s-\hat{s}|_{2}<$ $\frac{1}{2} c f(1-2 g)^{4}$. Applying this to (7.5) and again invoking inequality (7.4) we obtain

$$
\left|s^{\prime}-\hat{s}\right|_{2}>\frac{1}{2} c f(1-2 g)^{4}>\frac{\left(1+b_{k}\right)}{\sqrt{k}},
$$

which means that the alternative tree $\left(\mathcal{T}_{2}\right.$ or $\left.\mathcal{T}_{3}\right)$ is not a good tree. Thus, when event $E$ occurs, the set of good trees consists of $\mathcal{T}_{1}$ and no other tree (so that method $M$ will select the true tree, namely $\mathcal{T}_{1}$ ). Since event $E$ occurs with probability at least $1-\epsilon$ this verifies that $M$ satisfies condition (i) of Lemma 5.2.

We now establish that $M$ satisfies condition (ii). By Lemma 7.2, the probability that $M$ selects $\mathcal{T}_{1}$ (the true tree) is at least

$$
1 / 3\left(1-\exp \left(-b_{k} / 4\right)\right)
$$

(since the probability that $\mathcal{T}_{1}$ goes into the 'good' set is at least $1-\exp \left(-b_{k} / 4\right)$ and there are at most 3 good trees to select and so is bounded away from 0 ). Since $b_{k} \geq b_{1}$ for all $k$ and given the restriction placed on $b_{1}$, it follows that the probability that $M$ selects the true tree is at least $\delta$. This verifies that $M$ satisfies condition (ii) of Lemma 5.2. 
Acknowledgement We are indebted to Eva Czabarka for her comments on this paper. We also thank the anonymous reviewer for some helpful suggestions.

\section{References}

[1] Alon, N., and Spencer, J. H. (1992) The Probabilistic Method, John Wiley and Sons, New York.

[2] Bininda-Emonds, O. R. P., Brady, S. G., Kim, J., and Sanderson, M. J. (2001) Scaling of accuracy in extremely large phylogenetic trees. Pacific Symposium on Biocomputing 6 $547-558$.

[3] Colonius, H., and Schulze, H. H. (1981) Tree structures for proximity data. British Journal of Mathematical and Statistical Psychology, 34 167-180.

[4] Daskalakis, C., Mossel, E., Roch, S. (2005) Optimal Phylogenetic Reconstruction, to appear in STOC 2006, manuscript (ArXiv math.PR/0509575).

[5] Dekker, M. C. H. (1986) Reconstruction Methods for Derivation Trees, Master's Thesis, Vrije Universiteit, Amsterdam.

[6] Erdős, P. L., Steel, M. A., Székely, L. A., and Warnow, T. J. (1999) A few logs suffice to build (almost) all trees I, Random Structures and Algorithms 14(2) 153-184.

[7] Evans, W., Kenyon, C., Peres, Y., and Schulman, L. J. (2000) Broadcasting on trees and the Ising model. Adv. Appl. Prob. 10 410-433.

[8] Guindon, S., and Gascuel, O. (2003). A simple, fast, and accurate algorithm to estimate large phylogenies by maximum likelihood. Systematic Biology 52(5) 696-704.

[9] Lovász, L. (1979) Combinatorial Problems and Exercises, Akadémiai Kiadó, Budapest, and North-Holland.

[10] Mason, K. (1996) On a Matter of Uncertain Parentage, Honours III Project, University of Canterbury.

[11] Mossel, E. (1998) Recursive reconstruction on periodic trees, Random Structures Algorithms 13 81-97.

[12] Mossel, E. (2004) Phase transitions in Phylogeny Trans. Amer. Math. Soc. 356 (6) 23792404.

[13] Mossel, E. and Peres, Y. (2003) Information flow on trees. Annals of Applied Probability 13 (3) 817-844.

[14] Neyman, J. (1971) Molecular studies of evolution: a source of novel statistical problems. in Statistical Decision Theory and Related Topics, Gupta, S. S. and J. Yackel (eds), New York, Academic Press, 1-27.

[15] Rokas, A., and Carroll, S. B. (2005) More genes or more taxa? The relative contribution of gene number and taxon number to phylogenetic accuracy. Mol. Biol. Evol. 22 (5) 13371344.

[16] Semple, C., and Steel, M. (2003) Phylogenetics, Oxford University Press.

[17] Stamatakis, A.P., Ludwig, T., Meier, H. (2004). A fast program for maximum likelihoodbased inference of large phylogenetic tress. Proceedings of the 2004 ACM symposium on applied computing 197-201, ACM Press, New York, NY, USA.

[18] Steel, M. (1989) Distributions on Bicoloured Evolutionary Trees, PhD thesis, Massey University, Palmerston North, New Zealand.

[19] Steel, M., and Charleston, M. (1995) Five surprising properties of parsimoniously coloured trees. Bull. Math. Biol. 57(2) 367-375.

[20] Steel, M. A., Hendy, M. D., and Penny, D. (1998) Reconstructing phylogenies from nucleotide pattern frequencies - a survey and some new results, Discrete Applied Mathematics 88 367-396. 
[21] Steel, M. A., and Székely, L. A. (1999) Inverting random functions Annals of Combinatorics 3 103-113.

[22] Steel, M. A., and Székely, L. A. (2002) Inverting random functions II: explicit bounds for the discrete maximum likelihood estimation, with applications, SIAM J. Discrete Math. 15(4) 562-575. 